\title{
Verhandlungen des zweiten Kongresses des Nordischen Dermatologischen Vereines zu Stockholm den 5.-7. Juni 1913.
}

\author{
Nord. med. Arkiv 1913. Abt. II. Anhang. Stockholm 1914.
}

Aus der Fülle der mitgeteilten Krankheitsfälle und Berichten seien folgende hervorgehoben:

Rasch, C. Über Besniers Prurigo.

Zusammenbängender Bericht über Besniers Prurigo diatbèsique auf Grund der Arbeiten von Besnier, Hodara, Jadas obn, Sabouraud, Brocq und Jacquet. Der Vortrag hat mindestens 30 Fälle der Erkrankung gesehen. In vielen Fällen scheint eine Arsenbehandlung genützt zu haben, aber die besten Resultate hatte der Autor durch eine lange Zeit hindurch fortgesetzte laktovegetarische Diät.

Haslung, P. Über die Behandlung von Hautkrankheiten mit Kohlensäureschnee.

Das Hauptgebiet für diese Behandlung ist der Lupus erythematosus. Sitzungen von in der Regel 12 Sekunden mit kräftigem Druck. Bei den Naevi angiomatosi ist das Resultat unsicher, günstiger bei den Pigmentnaevis. Lupus vulgaris eignet sich nicht zur Gefrierbehandlung. Afzelius. Zur Frage von der Lokalisation der akuten strichförmigen Dermatosen.

Die mitgeteilten Fälle machen die Annahme am wahrscheinlichsten, daB auch die strichförmigen a $k$ ten Dermatosen metamerer Art seien. A. hält von den ausgestellten Hypothesen die Blaschkos für die plausibelste, daß die Segmentgrenzen auf Grund der embryonalen Verhältnisse bei der Entstehung derselben einen locus minori resistentiae bilden.

Moberg. Über das Analekzem und die Behandlung desselben.

M. verwendet die üblichen chemischen Mittel nach den Prinzipien der Ekzembehandlung, aber in Kombination mit Röntgenstrahlen und Hochfrequenzströmen.

Grön, Kr. Über Tätowierungen.

Kulturgeschichtlich hochinteressanter Vortrag, nicht zum kurzen Referat geeignet.

Bruusgaard, E. Von der akuten disseminierten Hauttuberkulose im Kindesalter.

Bericht über einen Fall, ein 4jähriges Mädchen, das neben einer Folliklis und einem Erythema induratum Bazin multiple verruköse Effloreszenzen aufweist. Die Dissemination der Hauttuberkulose hatte sich an Masern angeschlossen.

Jersild, 0. Vorläufige Mitteilung über eine neue abortive Behandlung der Gonorrhoe. 
542 Verh. des II. Kongr. des Nord. Derm. Vereines in Stockholm.

Empfehlung einer elektrolytischen Behandlung mit $1 \%$ Lösung von Kupfersulfat in der Urethra. Die nach den Angaben des Vortrag. sehr gute Wirkung beruht auf der Einfübrung vou Kupferionen in das Gewebe.

Der vorliegende Separatabdruck enthält noch weitere zahlreiche Berichte über Krankheitsfälle und Vorträge, die aber teilweise in unseren Deutschen Dermatologischen Zeitschriften schon publiziert sind.

Fritz Juliusberg (Posen).

\section{Verhandlungen der Sociedade Brasileira de Dermatologia.}

Sitzungen vom 7. April, 7. Mai, 28. Mai und 23. Juni 1913.

Terra, F. und Rabello, Ed. Das Ulcus tropicum und seine Behandlung.

Nach einer kurzen Besprechung der Literatur Mitteilung von 2 Fällen. Der 2. Fall, der ein Jahr lang lokal ohne Erfolg behandelt war, wurde geheilt durch die von $\nabla i a n n a$ und Arag Methode der intravenösen Injektionen von Tartarus stibiatus in $0.1 \%$ Lösung. Die Injektionen werden einen um den anderen Tag gegeben. Die Dosis ist 5-10 ccm. Nach 15 Injektionen Heilung. Keine unangenehmen Nebenwirkungen.

Terra, F. Ein Fall von Blastomykose.

23jähriger Arbeiter; Beginn der Erkrankung am 1. Nasenflügel, rascher Fortschritt auf die Schleimhaut von Nase und Wange. Ausgedehnter geschwüriger Zerfall, Vereiterung der benachbarten Lymphdrüsen. In den Ausstrichpräparaten von den Geschwüren und den vereiterten Lymphdrüsen Blastomyzeten in verschiedenen Stadien der Entwicklung. Keine genaueren Angaben. Jod nach 7 Monaten unter Kachexie. 1 Abbildung.

Diskussion. Vianna, E. Die Sektion ergab eine frische tuberkulöse Aussaat in den Iungen. In der Nebennierenkapsel, auf den Meningen und besonders reichlich in der Wand der Appendix grauweiße Knötchen, die als blastomykotische Veränderungen aufgefaßt wurden. In der Milz, den Nieren und in der Leber embolische Abszesse ebenfalls blastomykotischer Natur. In den mikroskopischen Präparaten aus den Lymphdrüsen reichlich Blastomy zeten. (Dem Ref. erseheint nacb dem vorliegenden Bericht der Charakter dieser Veränderungen als blastomykotisch nicht genügend begründet zu sein.)

Jut z, Ad. Allgemeinerkrankungen durch Blastomyzeten sind nicht selten. Die Art der Übertragung ist noch nicht aufgeklärt, es ist wahrscheinlich, daß Haustiere eine Rolle dabei spielen.

Rabello, Ed. Ein Fall von Botryomykose.

14jähriger Knabe. Seit einem Monat im AnschluB an ein Trauma gestielter, haselnußgroßer, gelappter, dunkelroter, leicht blutender Tumor 